\title{
Assessment of minimum permissible geometrical parameters of a near-to-eye display
}

\author{
Sergiy Valyukh and Oleksandr Slobodyanyuk
}

\section{Linköping University Post Print}

\section{Tweet}

N.B.: When citing this work, cite the original article.

Original Publication:

Sergiy Valyukh and Oleksandr Slobodyanyuk, Assessment of minimum permissible geometrical parameters of a near-to-eye display, 2015, Applied Optics, (54), 21, 6526-6533. http://dx.doi.org/10.1364/A0.54.006526

Copyright: Optical Society of America http://www.osa.org/

Postprint available at: Linköping University Electronic Press

http://urn.kb.se/resolve?urn=urn:nbn:se:liu:diva-120743 


\title{
Assessment of minimum permissible geometrical parameters of a near-to-eye display
}

\author{
Sergiy Valyukh,1,* Oleksandr Slobodyanyuk ${ }^{2}$ \\ ${ }^{1}$ Laboratory of Applied Optics, Department of Physics, Chemistry and Biology, \\ Linköping University, SE-581 83 Linköping, Sweden \\ 2 Department of Physics, Taras Shevchenko National University of Kyiv, Ukraine \\ *Corresponding author: serva@ifm.liu.se
}

Received Month X, XXXX; revised Month X, XXXX; accepted Month X, XXXX; posted Month X, XXXX (Doc. ID XXXXX); published Month X, XXXX

\begin{abstract}
Light weight and small dimensions are some of the most important characteristics of near-to-eye displays (NEDs) which consist of two basic parts: a microdisplay for generation an image and supplementary optics to be able to see the image. Nowadays, the pixels size of microdisplays may be less than $4 \mu \mathrm{m}$ that makes the supplementary optics as the major factor defining restrictions on a NED dimensions, or at least on the distance between the microdisplay and eye. The goal of the present work is to give answers on the following two questions: how small this distance can be in principle and what is the microdisplay maximum resolution that stays effective to see through the supplementary optics placed in immediate vicinity of the eye. To explore the first question, we consider an aberrations free magnifier that is the initial stage in elaboration of a real optical system. In this case the paraxial approximation and the transfer matrix method are ideal tools for simulation of light propagation from the microdisplay through the magnifier and the human eye's optical system to the retina. The human eye is considered according to the Gullstrand model. Parameters of the magnifier, its location with respect to the eye and the microdisplay, depth of field that can be interpreted as the tolerance of the microdisplay position are determined and discussed. The second question related to the microdisplay maximum resolution is investigated by using the principles of wave optics. (C) 2014 Optical Society of America

OCIS codes: (330.4060) Ophthalmic optics and devices; (080.3630) Geometric optics, lenses; (330.4060) Vision modeling;

http://dx.doi/org/10.1364/AO.99.099999
\end{abstract}

\section{Introduction}

During the last decade increased attention is paid to nearto-eye displays (NEDs) [1-9]. NEDs, which are usually associated with head mounted displays, are used solely as a hand-off information source in portable equipment for practical and leisure purposes. In ways that computers have come in all spheres of the human activities, NEDs have good chances to do the same. Numerous medical and military applications, telecommunication, transportation and game industry are only a few areas where NEDs are of inestimable value. Despite the dramatic advances in development of NEDs [1-9], expectations for NEDs with ergonomics comparable to eyeglasses have yet to be met [5].

Size and weight, which are very important parameters of a NED, depend critically not only on the microdisplay used but also on the optical system needed to transfer an image from the microdisplay onto a viewer's eye retina. In order to reduce the size of a NED and, as a result, to increase the viewer's comfort, several non-traditional optical design solutions for NEDs have been proposed. The majority of the proposed layouts, including much advertised in mass media Google Glass, consists of a tiny projector, several mirrors (semitransparent-mirrors) and lenses. The optical path from the image source to the cornea of the eye in this case can be around $10 \mathrm{~cm}$ that does not pose severe problems for seeing the image of the microdisplay. However, this technique cannot be applied for applications where the distance between the eye and the microdisplay has not to exceed a couple of centimeters or in some cases even much less. For example, these have been proposed NEDs that can be comfortably worn in the form of a contact lens [7-9]. The room accessible for supplemental optical system in such NEDs is restricted by several hundred micrometers. One of the possible solutions for such a situation is based on a microlens array serving as magnifier $[8,10,11]$. The same approach can be applied to a microdisplay incorporated into a lens of glasses. In contrast to a single objective lens, a microlens array is designed in such a way that every microlens enables one to see a certain small part of the image generated by the microdisplay. However, due to non-triviality of the problem, it is difficult to judge on potential of an objective lens or a microlens without a preliminary study. In particular, several questions arise: what is the limit for possible minimal geometrical sizes of the objective lens and microdisplay? Is it possible to see a microdisplay inbuilt into a contact lens? If yes, what is the maximum resolution of the microdisplay? 
In the present work, we make an effort to answer these questions by estimating the smallest possible distance between the microdisplay and the eye. As a first approximation, it is reasonable to apply the principles of Gaussian optics for finding paraxial image position provided by a centered optical system. Although the Gaussian theory cannot be used for entire estimation of an optical system quality, a simplified, though somewhat approximate, sketch of the action of the system and its elements can often be obtained without entering into the full intricacy of the techniques of optical design. Consideration of the principles of perfect imaging is the major stage in elaboration of real optical instruments. Subsequent accounting for aberrations as deviations from the ideal paraxial imaging may only worsen the optical system's data derived in paraxial approximation but not vice versa.

Applying the paraxial approximation will enable us to get the needed range of values concerning possible minimum geometrical parameters of NEDs and to conclude about the advisability of further application of more rigorous methods for lens design that has to be done in consideration of specifics of a particular NED. Inasmuch as the eye is an integral part of the optical system, it is necessary to trace the light rays from the microdisplay to the eye's retina. Optics of the human eye has been a subject of intensive investigations since development of optical instruments. Many of the proposrd models of the human eye often show good much with experimental data but still some discrepancies exist [12, 13]. There is no a universal model [13]. On the other hand, these discrepancies are associated with the wide angle viewing $[12,13]$ that is not considered in the paraxial approximation. Moreover, by studying a paraxial image formation, it is not necessary to take into account variations of the refractive indexes in the real human eye [12-15].

Imperfections in image formation or aberrations lie outside of the paraxial approximation. In real optical systems aberrations can be suppressed by numerous known methods like using of aspherical surfaces or compensating elements [16]. Certainly, design of any optical system has to be done taking in account specific features of image source (microdisplay) but this will not change essentially results obtained in the paraxial approximation. In the present paper we just draw a way to define a proper aspheric surface of the magnifier and consider the objective space in form of a curved surface that coincides with the surface of the microdisplay.

Since the pixels size of the microdisplay is compatible with the wavelength, the wave nature of light has to be applied for evaluation of the maximum reasonable resolution of the microdisplay. By doing so, we will define limitations of the pixels size.

\section{Transfer matrix of magnifier}

Propagation of light through a combined optical system that consists of objective lens, and eye sequentially placed on the optical axis can be traced with the transfer matrix method, or ABCD matrix method [17]. According to this approach, any optical element or system can be represented by the transfer matrix of $2 \times 2, \hat{M}$, that effects on a vector, the coordinates of which are the height, $y$, and the slope described by the angle $\theta$, of a ray at axial location:

$$
\left(\begin{array}{l}
y_{\text {out }} \\
\theta_{\text {out }}
\end{array}\right)=\left(\begin{array}{ll}
m_{11} & m_{12} \\
m_{21} & m_{22}
\end{array}\right)\left(\begin{array}{l}
y_{\text {in }} \\
\theta_{\text {in }}
\end{array}\right)=\hat{M}\left(\begin{array}{l}
y_{\text {in }} \\
\theta_{\text {in }}
\end{array}\right),
$$

where the subindexes in and out relate to incoming and outcoming rays.

The transfer matrix $\hat{M}$ of the combined optical system objective lens-eye is a product of the transfer matrixes of the eye, the translation distance $l$, the objective lens, and the translation distance a (Fig. 1), respectively,

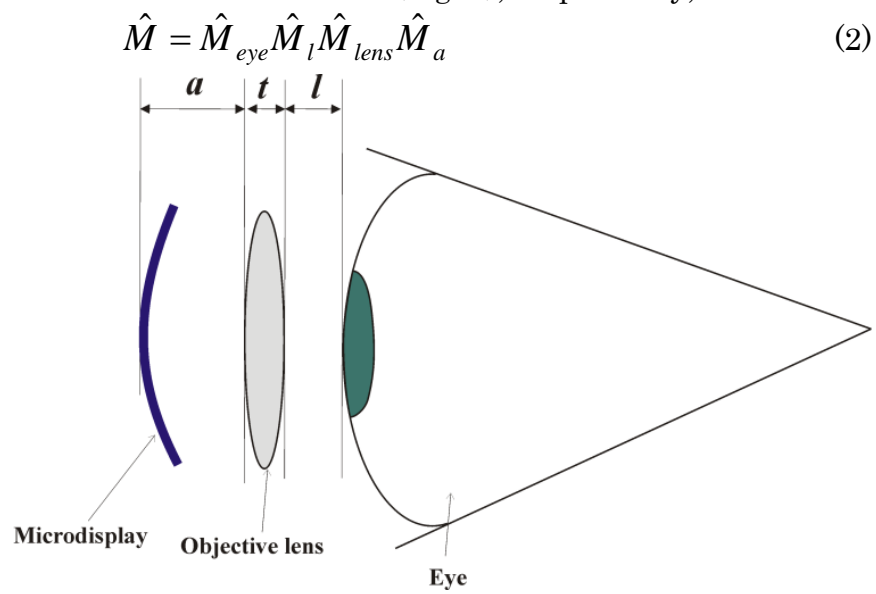

Fig. 1. Microdisplay and objective lens located near the eye.

It is wise to note that in practice the microdisplay can be flexible and be adjusted up to arbitrary curvature that is very important for reduction possible aberrations caused by the single singlet element.

The transfer matrixes of all optical elements that will be considered here can be expressed through the transfer matrixes of the translation distance $d$ and the spherical surface of the radius $R$ :

$$
\hat{M}_{\text {space }}=\left(\begin{array}{ll}
1 & d \\
0 & 1
\end{array}\right), \hat{M}_{\text {sphere }}=\left(\begin{array}{cc}
1 & 0 \\
\frac{n-n^{\prime}}{n^{\prime} R} & \frac{n}{n^{\prime}}
\end{array}\right) .
$$

Here a ray propagates from a material with the index $n$ to one with the refractive index $n^{\prime}$.

Thus, the transfer matrixes $\hat{M}_{l}$ and $\hat{M}_{a}$ have the same form as $\hat{M}_{\text {space }}$ but a or $l$ are set instead of $d$. The transfer matrix of a lens formed by two spherical surfaces of radiuses $R_{1}$ and $R_{2}$ as shown in Fig. 2 is the product:

$$
\hat{M}_{\text {lens }}=\hat{M}_{\text {sphere } R_{1}} \hat{M}_{\text {space } t} \hat{M}_{\text {sphere } R_{2}} \text {, }
$$

where $t$ is the center thickness of the lens.

On substituting (3) into (4), we get 
$\hat{M}_{\text {lens }}=\left(\begin{array}{cc}1+t \frac{n_{1}-n_{L}}{n_{L} R_{1}} & \frac{t n_{1}}{n_{L}} \\ \frac{n_{L}-n_{2}}{n_{2} R_{2}}+\frac{n_{1}-n_{L}}{n_{L} R_{1}}\left(\frac{n_{L}}{n_{2}}+\frac{t\left(n_{L}-n_{2}\right)}{n_{2} R_{2}}\right) & \frac{n_{1}}{n_{L}}\left(\frac{n_{L}}{n_{2}}+\frac{t\left(n_{L}-n_{2}\right)}{n_{2} R_{2}}\right)\end{array}\right),(5)$

where $n_{1}, n_{L}$ and $n_{2}$ are the refractive indexes of the materials as shown in Fig. 2.

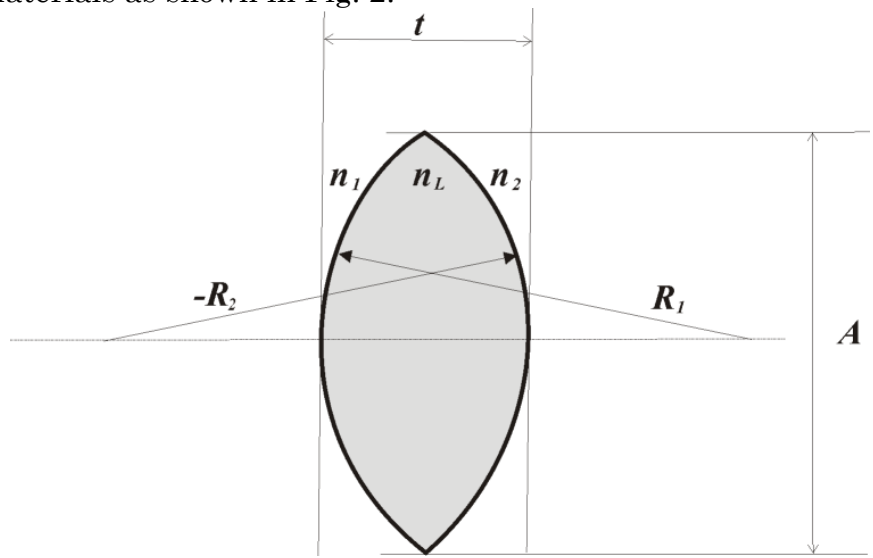

Fig. 2. Refractive lens restricted by a couple of spherical surfaces.

When $\theta_{i n}=0$, i.e. the incoming rays propagate parallel to the lens main optical axis, from (1) follows that the lens focal distance, $f$, is expressed through the transfer matrix element 21 as

$$
f=-\frac{1}{m_{21}} .
$$

Taking into account (5), we obtain

$$
f=\frac{n_{2} n_{L} R_{1} R_{2}}{n_{L}\left[\left(n_{L}-n_{1}\right) R_{2}-\left(n_{L}-n_{2}\right) R_{1}\right]+\left(n_{L}-n_{1}\right)\left(n_{L}-n_{2}\right) t} .
$$

Let us examine conditions under which $f$ reaches its minimum for a fixed linear aperture of the lens $(A)$. Expressing $t$ through $R_{1}, \quad R_{2}, \quad$ and $A$ as $t=R_{1}-\sqrt{R_{1}^{2}-(0.5 A)^{2}}-R_{2}+\sqrt{R_{2}^{2}-(0.5 A)^{2}}$, we found that $f$ has lowest value when the lens is symmetrical, i.e. $R_{1}=-R_{2}$, and $R_{1}, R_{2}$ are slightly larger than $A, n_{L}$ is as large as possible, while $n_{1}$ and $n_{2}$ are as small as possible. In addition, lower $A$ leads to lower $f$.

\section{Coupling with the optical system of the eye}

The human eye is the most complicated component for the simulation in the layout: microdisplay-magnifier-eye. A great variety of models describing the optics of the human eye, which itself poses a formidable challenge and is a complex optical system with evolutionary optimized design, have been proposed [12-15]. The variety of the models reflects the fact that all they are incomplete. Each of the models to a greater or lesser degree has own disagreement with the optical properties of the real human eye. Fortunately, the demands of the task considered here are eased by the fact that the paraxial approach and monochromatic light (no wavelength dispersion) can be applied for a sufficiently good estimation of the sizes of an optical system coupled with the eye. This make possible not to go into the biological or medical details of the eye and to utilize the model proposed by Allvar Gullstrand [12$15]$.

The geometry of the human eye is shown in Fig. 3. The light enters the eye through the cornea and then passes through the anterior chamber. The anterior chamber borders on the iris that serves as a diaphragm of variable diameter. After the iris, the lens capsule covering the crystalline lens is located. Behind the lens capsule, the light passes through the vitreous humor and is detected at the retina.

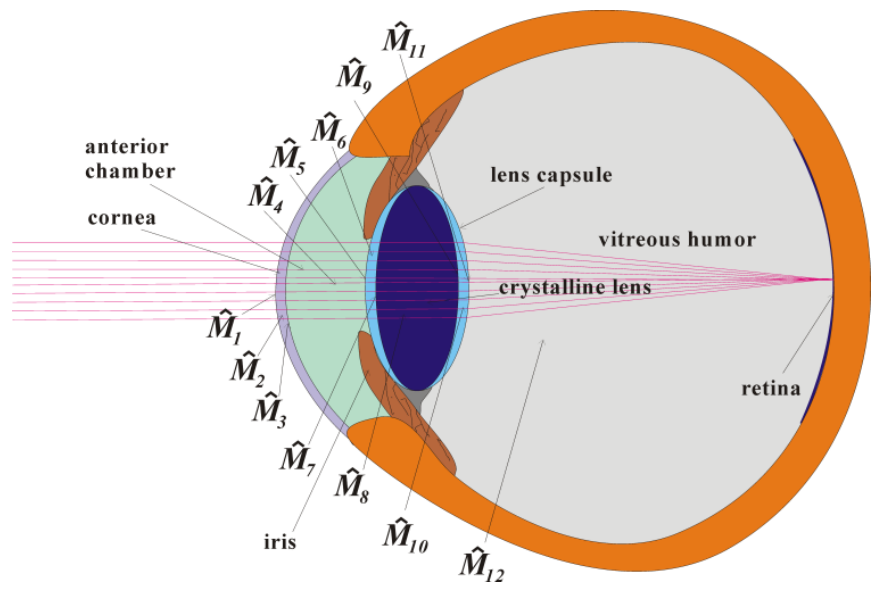

Fig. 3. Simplified scheme of the human eye and areas described by the matrixes $\hat{M}_{i}$

Thickness of the anterior chamber, as well as thicknesses and radiuses of the lens capsule and crystalline lens, are variable that allows to adjust the refractive power so that the eye can focus to an object a certain distance away. The geometrical parameters and refractive indexes of the eye's parts are summarized in Table 1. The parameters are given for two basic states of the eye: 1) the relaxed eye, when it focuses to infinity, and 2) the accommodated eye, when it focuses to the physiologically nearest point.

The transfer matrix of the schematic eye can be found as a product of the transfer matrixes of all 12 sequentially arranged optical elements as shown in Fig. 3:

$$
\hat{M}_{\text {eye }}=\prod_{i=12}^{1} \hat{M}_{i} \text {, }
$$

By composing the matrixes $\hat{M}_{i}$, where $i$ takes values from 1 to 12 , according to (3), inserting the corresponding data from Table 1 and after that substituting into (8), we obtain the numerical meaning of $\hat{M}_{\text {eye }}$ in the relaxed state

$$
\hat{M}_{\text {eye relaxed }}=\left(\begin{array}{cc}
0 & 17.0541 \\
-0.0439 & 0.6893
\end{array}\right),
$$

and in the accommodated state 


$$
\hat{M}_{\text {eye accomomdated }}=\left(\begin{array}{cc}
-0.1647 & 16.2839 \\
-0.0525 & 0.6774
\end{array}\right) .
$$

The dimensions of the matrixes elements are [mm] for $m_{12}$ and $[\mathrm{mm}]^{-1}$ for $m_{21}$. From (9), (10) and (6), we determine the optical refractive power of the relaxed eye as

Table 1. Gullstrand eye [12]

\begin{tabular}{lllllll}
\hline & Notation & \multicolumn{2}{l}{ Radius [mm] } & \multicolumn{2}{l}{ Thickness [mm] } & Refractive \\
\cline { 3 - 5 } & & Relaxed & Accommodated & Relaxed & Accommodated & 1.376 \\
\hline 1 & cornea & 7.70 & 7.70 & 0.50 & 0.50 & 1.336 \\
2 & anterior chamber & 6.80 & 6.80 & 3.10 & 3.10 & 1.386 \\
3 & front lens capsule & 10.0 & 5.33 & 0.546 & 0.6727 & 1.406 \\
4 & crystalline lens & 7.911 & 2.655 & 2.419 & 2.655 & 1.386 \\
5 & rear lens capsule & -5.76 & -2.655 & 0.635 & 0.6725 & 1.336 \\
6 & Vitreous humor & -6.00 & -5.33 & 17.185 & 16.80 & \\
7 & Retina & -17.2 & -17.2 & & & \\
\hline
\end{tabular}

with the experimental results $[12,13]$.

\section{Depth of field}

Although a lens or an optical system consisting of few lenses can precisely focus at only one distance simultaneously, the sharpness decreases gradually on each side of this distance. To obtain a good image quality in a NED, it is necessary to match the image source (microdisplay) with the objective plane of the optical system of the NED. The tolerance in matching the image with the objective plain can play a critical role for practical use of the NED. Thus, a low tolerance, e.g. several micrometres, complicates the manufacturing process and causes difficulties in future exploitation.

The range of distances within which any point has an acceptably sharp image is the lens (or the optical system) characteristic known as depth of field (DOF) [18]. DOF can be defined as the difference between coordinates of the nearest, $a_{N}$, and farthest, $a_{F}$, objects in a scene for which images are still sharp

$$
D O F=a_{F}-a_{N} .
$$

By applying the transfer matrix method described above, it is possible to determine DOF of the combined system objective lens -eye. Assume that the sharp image of the microdisplay is observed when the distance between the microdisplay and the objective lens is a (Fig. 4). If to change this distance towards $a_{N}$ or in the opposite direction to $a_{F}$, the image of any point belonged to the microdisplay will transform to a blur, the radius of which increases and reaches its maximum when the distance is $a_{N}$ or $a_{F}$. As a criterion for the blur maximum value, it is reasonable to take the size of the sharp image of the microdisplay pixel.

Let the main optical axis of the optical system passes through the center of a pixel of the height $h$ and its image on the eye retina that has the height $H$. By using (1), we can write for the ray propagating from the pixel's edge parallel to the main optical axis:
43.9 dioptres and the optical power of the accommodated eye as 52.5 dioptres. These values are in a good agreement 
or

$$
m_{12}=0
$$

Eq.(2) gives

$$
m_{12}=m_{11}^{\text {eye }} m_{12}^{\text {lens }}+a\left(m_{11}^{\text {eye }} m_{11}^{\text {lens }}+\left(m_{12}^{\text {eye }}+m_{11}^{\text {eye }} l\right) m_{21}^{\text {lens }}\right)+\left(m_{12}^{\text {eye }}+m_{11}^{\text {eye }} l\right) m_{22}^{\text {lens }} .
$$

Combining (18) and (19), we have

$$
a=-\frac{m_{11}^{\text {eye }} m_{12}^{\text {lens }}+\left(m_{12}^{\text {eye }}+m_{11}^{\text {eye }} l\right) m_{22}^{\text {lens }}}{m_{11}^{\text {eye }} m_{11}^{\text {lens }}+\left(m_{12}^{\text {eye }}+m_{11}^{\text {eye }} l\right) m_{21}^{\text {lens }}} .
$$

Taking into account (9), it is possible to write for the relaxed eye

$$
a=-\frac{m_{22}^{\text {lens }}}{m_{21}^{\text {lens }}},
$$

or, according to (6),

$$
a=m_{22}^{\text {lens }} f .
$$

In order to find DOF, it is necessary to express $a_{N}$ and $a_{F}$ through the parameters of the considered optical system. By using (2), the equation for $a_{N}$ is

$$
\left(\begin{array}{c}
\frac{H}{2} \\
\theta_{\text {out }}
\end{array}\right)=\hat{M}\left(\begin{array}{c}
0 \\
\theta_{\text {in } \max }
\end{array}\right) \text {, }
$$

where $a_{N}$ is included instead of $a$, and $\theta_{\text {in max }}$ is the angle of the maximum deviation of the ray from the main optical axis.

Assuming that $\theta_{\text {in max }}$ is restricted by the lens aperture $A$, we can express: $\tan \theta_{\text {in } \max }=\frac{A}{2 a_{N}+t}$, where values of the tangents or directional derivatives has to be substituted into (23) instead $\theta$ in max and $\theta_{\text {out }}$, if $A$ is large and the trigonometric functions cannot be expressed as linear functions of the angles. From (23),

$$
2 A m_{12}=\left(2 a_{N}+t\right) H .
$$

Taking into account (19) and (24), we find

$$
a_{N}=\frac{H t-2 A\left(m_{11}^{\text {eye }} m_{12}^{\text {lens }}+\left(m_{12}^{\text {eye }}+m_{11}^{\text {eye }} l\right) m_{22}^{\text {lens }}\right)}{2 A\left(m_{11}^{\text {eye }} m_{11}^{\text {lens }}+\left(m_{12}^{\text {eye }}+m_{11}^{\text {eye }} l\right) m_{21}^{\text {lens }}\right)-2 H} .
$$

In a similar way, it is possible to find $a_{F}$ for which $\frac{H}{2}$ in (23) must be replaced to $-\frac{H}{2}$. By doing so, we get

$$
a_{F}=-\frac{H t+2 A\left(m_{11}^{\text {eye }} m_{12}^{\text {lens }}+\left(m_{12}^{\text {eye }}+m_{11}^{\text {eye }} l\right) m_{22}^{\text {lens }}\right)}{2 A\left(m_{11}^{\text {eye }} m_{11}^{\text {lens }}+\left(m_{12}^{\text {eye }}+m_{11}^{\text {eye }} l\right) m_{21}^{\text {lens }}\right)+2 H} \text {. }
$$

After that, DOF is found from (11) with taking into account (25), (26), (13) and (14).

For the relaxed eye (9), when $D O F$ has the relatively simple expression in comparison with the accommodated eye, we have

$$
D O F=\frac{A h\left(2 m_{22}^{\text {lens }} f+t\right)}{A^{2}-h^{2}} .
$$

In estimation of DOF of a NED, two aspects have to be recognized: 1) the NED is designed to project the microdisplay image to a certain distance from the eye, e.g. $25 \mathrm{~cm}$ or infinity. DOF can be defined for the eye that is always focused on a designed distance. This is especially important when the NED is an augmented reality (or mixed reality) display [9-11]. For example, a driver can simultaneously get information generated by microdisplay and observe distant objects without change of eye accommodation, i.e. without losing control over the traffic situation. DOF obtained in (27) is relevant namely to such a case. 2) The eye can change accommodation to focus on the microdisplay image. In this case, DOF must be defined as the difference between $a_{F}$ found for the relaxed eye and $a_{N}$ found for the accommodated eye. The result will be the same if to add (27) to the difference between two $a_{N}$ determined for the accommodated (10) and relaxed (9) states.

\section{Minimum size of the pixel}

One of the important characteristics of the magnifier considered here, as well as any imaging system, is its optical resolution that describes the ability of the lens (or the imaging system) to resolve details in the object that is being imaged. According to the Rayleigh criterion [16], an objective lens having a circle aperture is able to distinguish two adjacent points in the object separated by a distance $h_{\min }$, as small as

$$
h_{\min }=\frac{0.61 \lambda}{n \sin \alpha},
$$

where $\lambda$ is the wavelength, $\alpha$ is the half of the angular aperture of the lens. Expressing $\sin \alpha$ through $f$ and $A$ we can obtain

$$
h_{\min }=\frac{0.61 \lambda \sqrt{A^{2}+4 f^{2}}}{n A} .
$$

In terms of the display, $h_{\min }$ can be considered as the minimum size of the display pixel. In order to find $h_{\min }$ that corresponds to the minimum value of the ratio $f / A$ known as the F-number, let us express $t$ through $A$ as $t=\zeta A$, where $\zeta$ is some coefficient. Assuming that $-R_{2}=R_{1}=R, n_{1}=n_{2}=n$ and substituting $R$ expressed through $A$ and $t \quad\left(R=\frac{A^{2}}{4 t}+\frac{t}{4}\right)$ into (7), we derive

$$
\frac{f}{A}=\frac{n n_{L}\left(1+\zeta^{2}\right)^{2}}{8 \zeta\left(n_{L}-n\right)\left(n_{L}+\left(2 n-n_{L}\right) \zeta^{2}\right)} .
$$

The ratio in (30) reaches its minimum for $n_{L}<2 n$ when

$$
\zeta=\sqrt{\frac{\sqrt{9 n^{2}-16 n n_{L}+8 n_{L}^{2}}-3\left(n_{L}-n\right)}{2 n-n_{L}}} .
$$

Substituting (31) into (30) and then into (29) gives $\zeta=0.68$ and $h_{\min }$ is around $1.03 \lambda$ when $n=1$ and $n_{L}=1.5$. For these parameters, the $\mathrm{F}$-number is also equal to 0.68 .

\section{Results and discussion}


Analytics derived in the previous sections enables us to obtain quantitative values of the magnifier parameters that will give a more or less clear picture regarding possible geometrical sizes and resolution of a NED to be utilized. The above results were derived under the assumption that the lens gives the perfect image of an object, i.e. there are no aberrations. However, a spherical refractive surface is associated with spherical aberrations [16, 18], which start to be noticeable when the light beam is sufficiently wide (this aberration is proportional to the third power of the zonal radius $\rho$ ). Exploration of the dependence of the aberrations on the aperture of the light beam brings us to the following result: the aberrations of a thick spherical lens with $t$ varying between $0.5 A$ and $A$ are sufficiently small if the diameter of the light beam does not exceed $\frac{t}{n_{L}}$. If so, by restricting the beam with an aperture stop of diameter $\frac{t}{n_{L}}$, we will obtain the F-number ranged between $1.02(t=A)$ and 2.04 $(t=0.5 A)$. In this case $h_{\min }$ lays between $1.39 \lambda$ and $2.57 \lambda$ that is still less than the sizes of pixels used in the microdisplays. There are known the following methods for supressing spherical, as well as other type of geometrical aberrations, that consist in 1) adding additional optical elements having aberration of diferent sings 2) development of aspherical surfaces and 3) using gradient optics On the other hand, the spherical aberrations can be essentially reduced due to applying either an objective lens consisted of several singlets or an aspherical lens [16-18] whose surface profiles can be considered as portions of spheres only for small angles between rays and the optical axis.

Since pixels of a microdisplay have usually a rectangle shape, the most effective shape of the microlenses in the microlens array is a rectangle too. For determining $h_{\min }$ in this case, it is necessary to replace coefficient 0.61 in (28) and (29) by 0.5 [18]. Another feature that must be taken into account is that $A$ must be much larger $\lambda$. Otherwise, the light flow passing the microlens will be governed by diffraction rather than by the focusing properties of this microlens.

Let us determine the minimum distance between the eye and the microdisplay that can be achieved. For the eye's relaxed state, the microdisplay must be placed in the focal plane of the magnifier. The minimum distance in this case as it will be demonstrated below is slightly higher than for the accommodated eye. When this occurs, 1 can take any value and the minimum distance is defined as the sum $t+a$ that, in turn, depends on $A, n_{L}$, and $n$. The desired case is a small value of the sum $t+a$ and sufficiently large $A$. Evidently, such a situation happens when the ratio $\frac{A}{t+a(A)}$ reaches its maximum. The curves in Fig. 5 show the behavior of $\frac{A}{t+a(A)}$ versus $A$ for $t$ equal to $0.1 \mathrm{~mm}$, $0.5 \mathrm{~mm}, 1 \mathrm{~mm}, 2 \mathrm{~mm}$ and $3 \mathrm{~mm}, n_{L}=1.5$ and $n=1$. By differentiating $\frac{A}{t(A)+a(A)}$ with respect to $\mathrm{A}$ and by setting the obtained result to zero, we find that the maximum is reached when $t=0.497 A$. The lens focal distance in this case equals $0.722 A$ and $t+a=1.029 A$. If to take $A=9 \mathrm{~mm}$ that corresponds to the maximum value of an adult human eye pupil diameter at dark, the minimum distance between the eye and the microdisplay is around $9.4 \mathrm{~mm}$.

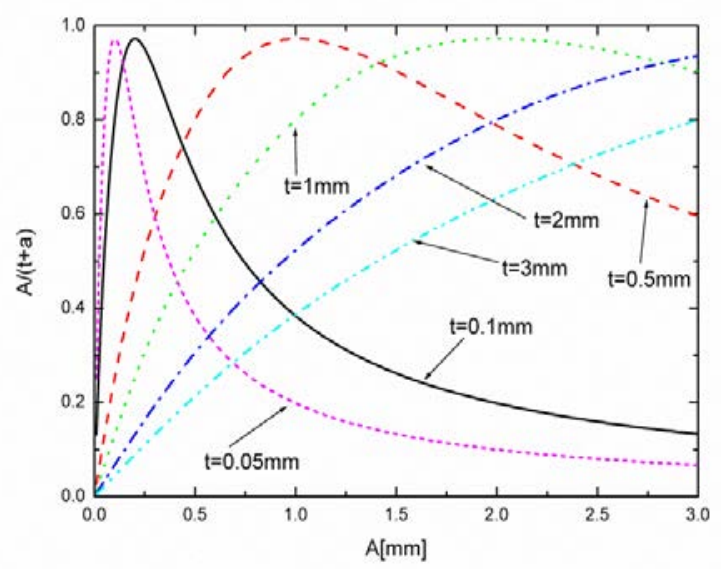

Fig. $5 A /(a+t)$ versus $A$

In order to decrease the distance between the microdisplay and cornea $(t+a)$, e.g. for a display incorporated into a contact lens $[7,8,9]$, it is necessary to decrease $A$. One of the proposed solutions is based on a microlens array where each microlens coupled with one or several pixels $[10,11]$. In this case, for example, a microlens of size $A=30 \mu \mathrm{m}$ [19] gives $t+a$ as small as $31 \mu \mathrm{m}$.

The lens parameters $(t, f)$ enabling achievement of the minimum distance between the eye and the microdisplay $(t+a)$, as well as the minimum value of the pixel $\left(h_{\min }\right)$, for several values of $n$ and $n_{L}$ are summarized in Table 2 .

Table 2. Parameters of the lens that provide the minimum distance between the eye and microdisplay

\begin{tabular}{cccccc}
\hline$n$ & $n_{L}$ & $h_{\min }$ & $t$ & $t+a$ & $f$ \\
\hline 1 & 1.33 & $1.32 \lambda$ & $0.582 A$ & $1.328 A$ & $0.996 A$ \\
1 & 1.5 & $1.03 \lambda$ & $0.497 A$ & $1.029 A$ & $0.722 A$ \\
1 & 1.7 & $0.88 \lambda$ & $0.430 \mathrm{~A}$ & $0.849 \mathrm{~A}$ & $0.564 \mathrm{~A}$ \\
1.33 & 1.5 & $0.78 \lambda$ & $0.757 \mathrm{~A}$ & $2.606 \mathrm{~A}$ & $2.215 \mathrm{~A}$ \\
\hline
\end{tabular}

According to the presented results, the values of $h_{\min }$ are less than sizes of the pixels in microdisplays. This fact makes possible to use microdisplays with high resolution even for a NED incorporated in a contact lens. For example, if the pixel is around $5 \mu \mathrm{m}$, the microdisplay coinciding with the size of the eye's pupil can have resolution more than $1000 \times 1000$ pixels. In order to see both the microdisplay located immediate vicinity of the eye and objects from ambient, it is necessary to have a switchselectable lens. Liquid crystal (LC) lenses are most suitable for such purposes [19-22]. Although they cannot compete with refractive lenses in low F-number, LC lenses, nevertheless, are suited for a microlens array for a contact lens $[10,11]$.

Expression (29) enables us to evaluate $h_{\min }$ for a liquid crystal microlens. If, for example, the microlens of thickness $d$ has linear aperture $6 d$, and the LC birefringence $\Delta n$ is 0.25 , then the maximum focus distance is around $18 d$ [18]. Substituting $A=6 d$ and $f=18 d$ to (29), we obtain $h_{\min }$ equal to $3.71 \lambda$. 
In designing optical instruments a greater accuracy than that given by Gaussian optics is needed. More precise determination of the light path may be done by algebraic analysis, considering the higher order terms in the expansion of the characteristic function [16]. Another way for determine of the path of the light rays is the ray tracing, where the consideration is based on elementary geometry with successive application of the law of refraction [16]. As an example, the ray tracing in the optical layout microdisplay - magnifier (objective lens) the eye is shown in Fig. 6 where the mesh interval is $1 \mathrm{~mm}$. The calculations were carried out accordingly Eqs (4) and (8) for the eye parameters presented in Table 1, the refractive indexes of the magnifier and the ambient are 1.5 and 1 , respectively, the lens aperture is $7.7 \mathrm{~mm}$, $t=3.82 \mathrm{~mm}$, and $a=3.95 \mathrm{~mm}$. The eye is in the relaxed state, i.e. the image of the microdisplay is projected to infinity. Although, we used the models adapted for the paraxial approach, as seen from Fig. 6 , the ray tracing with not wide beams gives an insight into image formation on the retina and the geometries of the microdisplay and magnifier, as well as their locations with respect to the eye. Specifically, from Fig. 6 follows that to obtain a better image quality, the microdisplay must have a curved shape that is easy achieved with a flexible displays.

From the results presented in Fig. 6, it is possible to estimate restrictions of the approach applied. In this particular example, it is seen that the single lens having spherical surfaces can project an image with relatively small abberations when the angle between the main optical axis and the rays does not exceed approximately 11 degrees. The viewing angle is equal approximately to $2 \cdot 14.5=29$ degrees. The image source has to be suited on a curved surface as shown in Fig. 6. The curved surface of the object space that must be optically conjugated to the image space situated on the retina, as well as wideness of the beams divergence was estimated from visual analysis in the program used. The main criterion of this analysis was the focusing of a beam on the retina to the light spot of acceptable size. In the considered example, the light spots of the beams from point sources are around $80 \mu \mathrm{m}$. The obtained restrictions are caused not only by the spherical surfaces of the magnifier but also by the Gullstrand model that are suitable for the paraxial approach.

Nevertheless, despite of the restrictions of the Gullstrand model and the spherical profile of the lens, from Fig. 6 follows that it is possible to see an image located at less than $8 \mathrm{~mm}$ from the eye. The size of the microdisplay in this case has to be around $5 \mathrm{~mm}$. To avoid aberrations, a diaphragm of diameter approximately $2 \mathrm{~mm}$ that is around $26 \%$ of the magnifier aperture has to be installed between the eye and lens. For tracing with wide beams, it is necessary to apply a more realistic model of the eye where the gradient changes of the refractive indexes in the eye and aspheric profiles of the interfaces between different parts of the eye are taking into account [13]. The surfaces of the magnifier in this case must be aspheric [16]. By doing so, one will obtain wide viewing angles than that mentioned above.

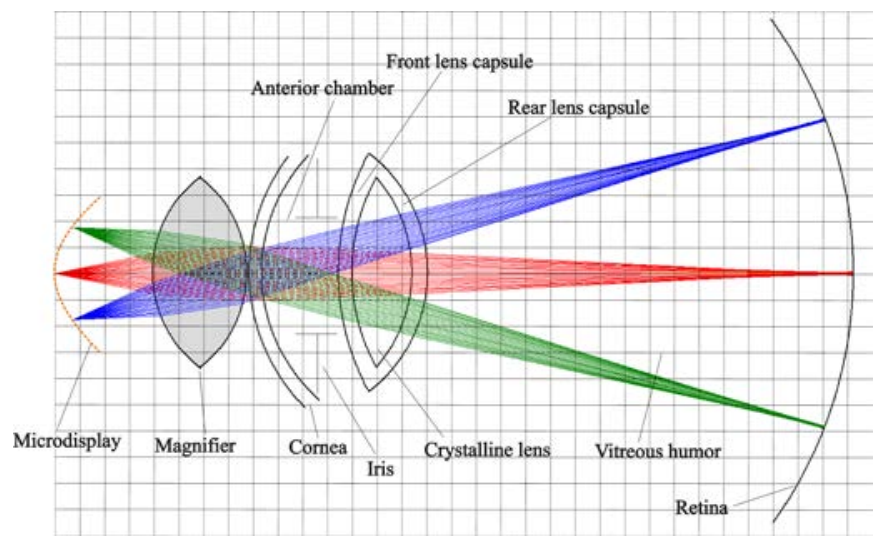

Fig. 6. Ray tracing in the optical layout: microdisplay magnifier (objective lens) - the human eye (Gullstrand model). The calculations are made with our internal software GOPTICS. The eye model parameters are taken from Table 1 . The grid step is $1 \mathrm{~mm}$.

By using Eq. (25-27), it is possible to estimate $D O F$ that can be interpreted as the tolerance of the distance between the magnifier (or the array of magnifiers) and the microdisplay. Whereas the distance between the eye and the microdisplay decreases with increasing the F-number of the magnifier, the situation for DOF is inverse. To gain an impression on the values of DOF at optimal parameters of the lens, we calculated dependences of $D O F / h$ versus the ratio $A / h$ for the lens with $t=0.497 \mathrm{~A}, n_{L}=1.5, n=1$. The obtained results are plotted in Fig. 7. The calculations were done for the following cases: 1) the eye is always relaxed, $h=10 \mu \mathrm{m}, 2) a_{F}$ is defined for the relaxed eye and $a_{N}$ for the accommodated eye, $h=10 \mu \mathrm{m}$, and 3) $a_{F}$ is defined for the relaxed eye and $a_{N}$ for the accommodated eye, $h=100 \mu \mathrm{m}$. From the presented results, we see that DOF has the same order of magnitude as $h$.

As expected, the increasing of DOF due to selfaccommodation of the eye is as higher as longer the focus distance of the magnifier. This is because the fraction of the optical refractive power of the eye becomes higher with respect to the optical refraction power of the magnifier. In the case when $A$ is $7.7 \mathrm{~mm}$ and $t+a$ is $7.9 \mathrm{~mm}$, the distance within which the eye can see clearly the microdisplay due to self-accommodation varies within $0.3 \mathrm{~mm}$.

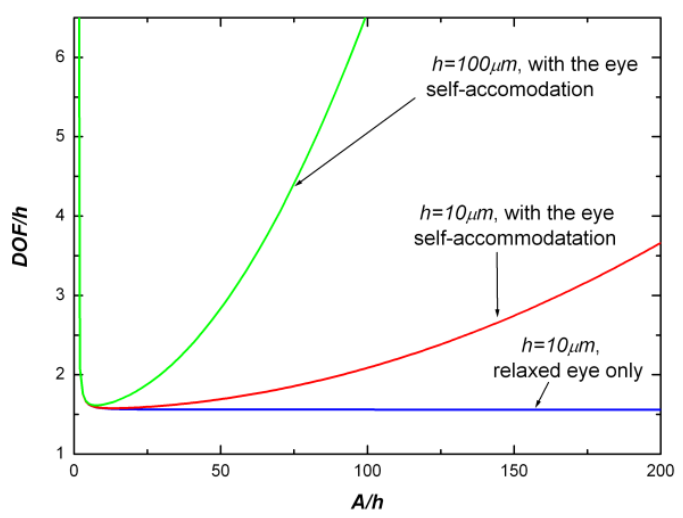


Fig. 7. DOF/ $h$ versus $A / h$

In summary, although we have mostly considered the customary refractive lens, the many results obtained in this work can be applied for other types of lens, e.g. gradient index (GRIN) lenses [18,20-22]. GRIN lenses based on liquid crystals are tuneable and can be driven by low voltage (up to $1 \mathrm{~V}$ ) [20-22]. By using this feature, it is possible to increase essentially functionality of NED. In particular, LC lenses enable one an easy way to see simultaneously both an image produced by a display and objects from the ambient.

\section{Conclusion remarks}

The minimum distance between the microdisplay of a NED and the eye is defined mainly by the size of the optical system of the NED. In the present work, we estimate how small this distance can be. The considered objective lens is an aberration free convex lens acting as a magnifier that is the principal stage in elaboration of a real objective lens. The found minimum distance between the eye and microdisplay is of order of linear aperture of the magnifier. In other words, a smaller linear aperture corresponds to the smaller distance. For example, a lens having linear aperture $9 \mathrm{~mm}$ (the diameter of the pupil of an adult human eye at dark) provides the minimum distance between the eye and microdisplay less than $1 \mathrm{~cm}$. This means that the microdisplay can be incorporated into ordinary glasses. The use of the Fresnel lens enables reducing the minimum distance approximately twice. For a microlens array inbuilt into a contact lens, this distance can be less than $0,1 \mathrm{~mm}$.

Depth of field of the magnifier coupled with the eye, which can be interpreted as the accessible tolerance in position of the microdisplay, depends on the F-number of the lens and the pixels size. If the lens aperture overlaps more than ten pixels, DOF is of order of magnitude of the pixel size for the relaxed eye. The self-accommodation of the eye increases DOF by hundreds micrometres for magnifiers with linear aperture of several millimetres and does not make essential effect for a microlens.

The found minimum pixels size, which depends on the Fnumber and on refractive index of the lens, as well as on the refractive index of the medium surrounding the lens, varies from $0.78 \lambda\left(n=1.33, n_{L}=1.5\right)$ up to $3.71 \lambda$ for a $\mathrm{LC}$ microlens. Assuming $\lambda=0.75 \mu \mathrm{m}$, the minimum possible pixel sizes does not exceed $2.8 \mu \mathrm{m}$ that is less than the pixels sizes of the reported microdisplays.

The obtained results can be used for further estimations of a single objective lens as well as a microlens in a lens array for NED.

\section{References}

1. H. Mukawa, K. Akutsu, I. Matsumura, S. Nakano, T. Yoshida, M. Kuwahara, K. Aiki, "A full-color eyewear display using planar waveguides with reflection volume holograms," J. SID, 17, 185-193, (2009).

2. M. B. Spitzer, P. D. Aquilino, R. W. McClelland, M. H. Olson, N. M. Rensing, C. A. DiMarzio, P. M. Zavracky, A. Lemoncelli, J. Hilliar, "Optical
Approaches To Incorporation of Displays Within Eyeglasses," Proc. SID Symp. Digest, 29, 10071009 (1998).

3. T. Järvenpää, V. Aaltonen, "Compact near-to-eye display with integrated gaze tracker," Proc. of SPIE 7001, 700106, (2008).

4. M. Pölönen, J. Häkkinen, "Near-to-Eye DisplayAn Accessory for Handheld Multimedia Devices: Subjective Studies," J. DISP. Tech, 5, 358-367 (2009).

5. M. Pölönen, T. Järvenpää, J. Häkkinen, "Comparison of Near-to-Eye Displays: Subjective Experience and Comfort”, J. Disp. Tech., 6, 27-35, (2010).

6. D. Lanman, D. Luebke, "Near-Eye Light Field Displays," ACM Trans. Graph. 32, 6, 220 (2013).

7. J. De Smet, A. Avci, R. Beernaert, D. Cuypers, H. D. Smet, "Wrinkle formation in conformable liquid crystal cells for use in a contact lens display", Proc. of IDW'11, 1203-1206. (2011)

8. A. R. Lingley, M. Ali, Y. Liao, R. Mirjalili, M. Klonner, M. Sopanen, S. Suihkonen, T. Shen, B. P. Otis, H. Lipsanen, B. A. Parviz, "A Singe-Pixel Wireless Contact Lens Display", Journal of Micromechanics and Microengineering, 21, 125014, (2011).

9. B. Furht (ed.), Handbook of Augmented Reality, DOI 10.1007/978-1-4614-0064-6, (Springer, 2011).

10. S. Valyukh, J. D. Smet, O. Slobodyanyuk, H. De Smet, "A Liquid-Crystal-Lens-Array Based Projection System for Near Eye Displays," Proc. 19th IDW/AD, 1305-1308, (2012).

11. S. Valyukh, O. Slobodyanyuk, "Objective LC Lens Array for a Near-to-Eye Display," SID Symp. Digest Technical Papers, 44, 891-894, (2013).

12. H. Gross, F. Blechinger, B. Achtner, Handbook of Optical Systems, Vol. 4, Survey of Optical Instruments, (Wiley, 2008).

13. R. Navarro, "The Optical Design of the Human Eye: a Critical Review," J Optom. 2, pp. 3-18, (2009).

14. W. N. Charman, "The Charles F. Prentice Award Lecture 2005: Optics of the Human Eye: Progress and Problems," Optometry and Vision Science, Vol. 83, No. 6, PP. 335-345 (2006).

15. W. N. Charman, "Optics of the eye," chap. 24 in Handbook of Optics; Vol. 1, M. Bass, editor in chief, 2nd ed., (McGRAW-HILL, New York, 1995).

16. M. Born, E. Wolf, "Principles of Optics: Electromagnetic Theory of Propagation, Interference and Diffraction of Light," $7^{\text {th }}$ ed, (Pergamon press, 1999)

17. A. Gerrard \& J.M. Burch,. "Introduction to Matrix Methods in Optics," (Dover 1994).

18. F. Träger (ed.), Handbook of lasers and optics, (Springer, 2007). 
19. P. Ruffieux, T. Scharf, I. Philipoussis, H.P. Herzig, R. Voelkel, K. J. Weible, "Two step process for the fabrication of diffraction limited concave microlens arrays," Opt. Exp. 16, 19541-19549 (2008).

20. S. Valyukh, I. Valyukh, V. Chigrinov, H.S. Kwok, H. Arwin, "Liquid crystal light deflecting devices based on non-uniform anchoring," Appl. Phys. Lett. 97, 231120 (2010).

21. S. Valyukh, V. Chigrinov, H.S. Kwok, H. Arwin, "On liquid crystal diffractive optical elements utilizing inhomogeneous alignment," Optic. Expr. 20, 15209 (2012).

22. S. Valyukh, V. Chigrinov, H. S. Kwok, "A liquid crystal lens with non-uniform anchoring energy," SID Symp. Digest Technical Papers, 39, 659-662 (2008) 


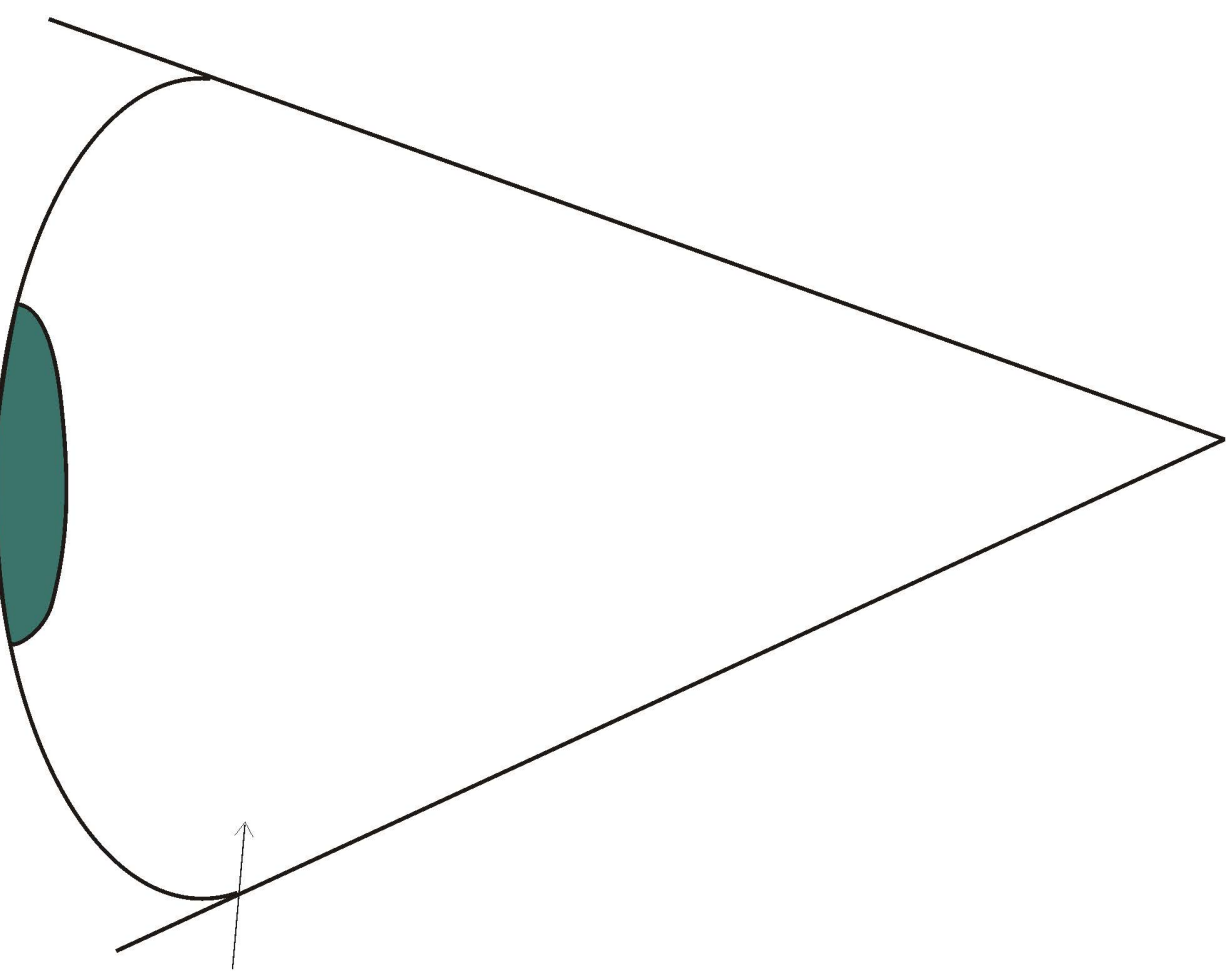





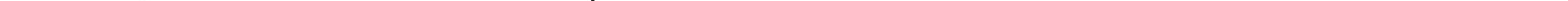




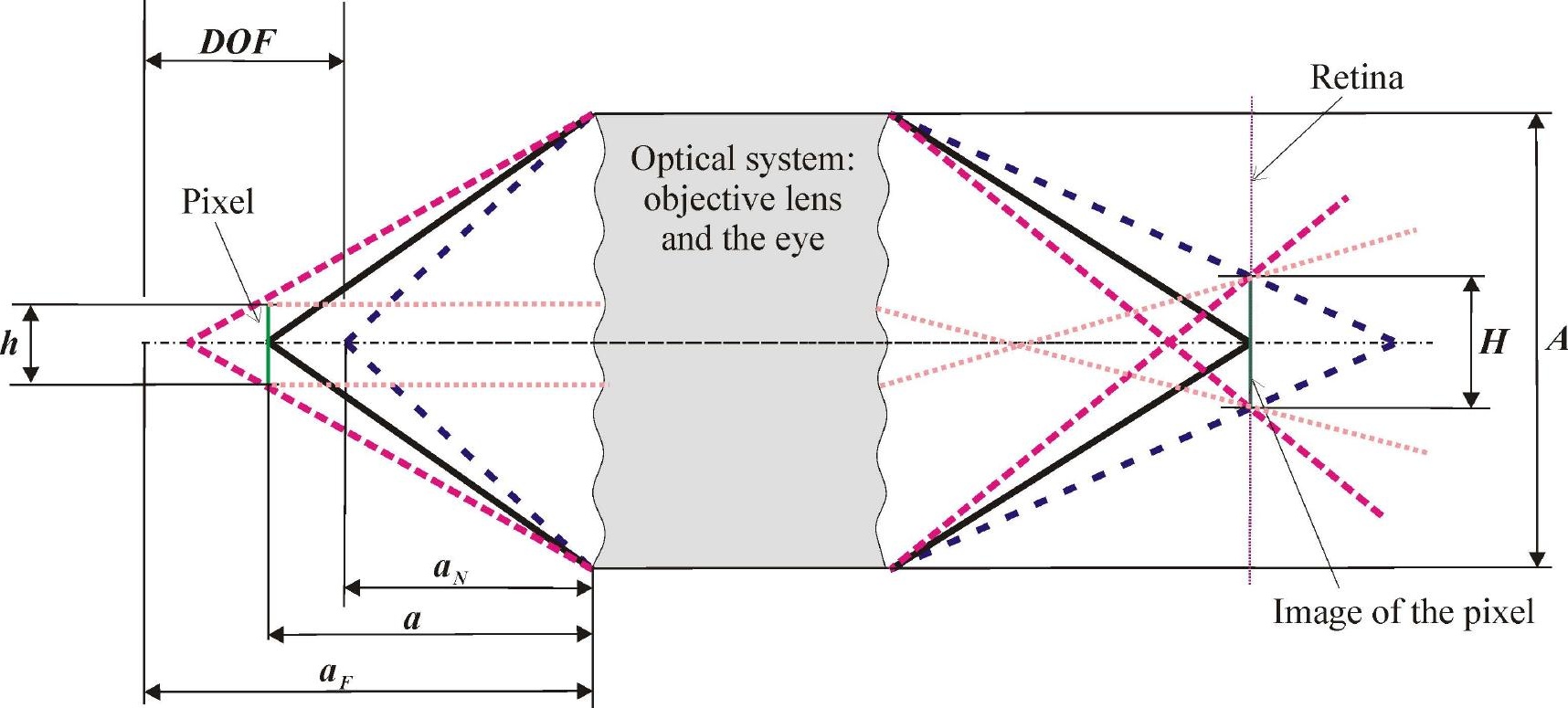




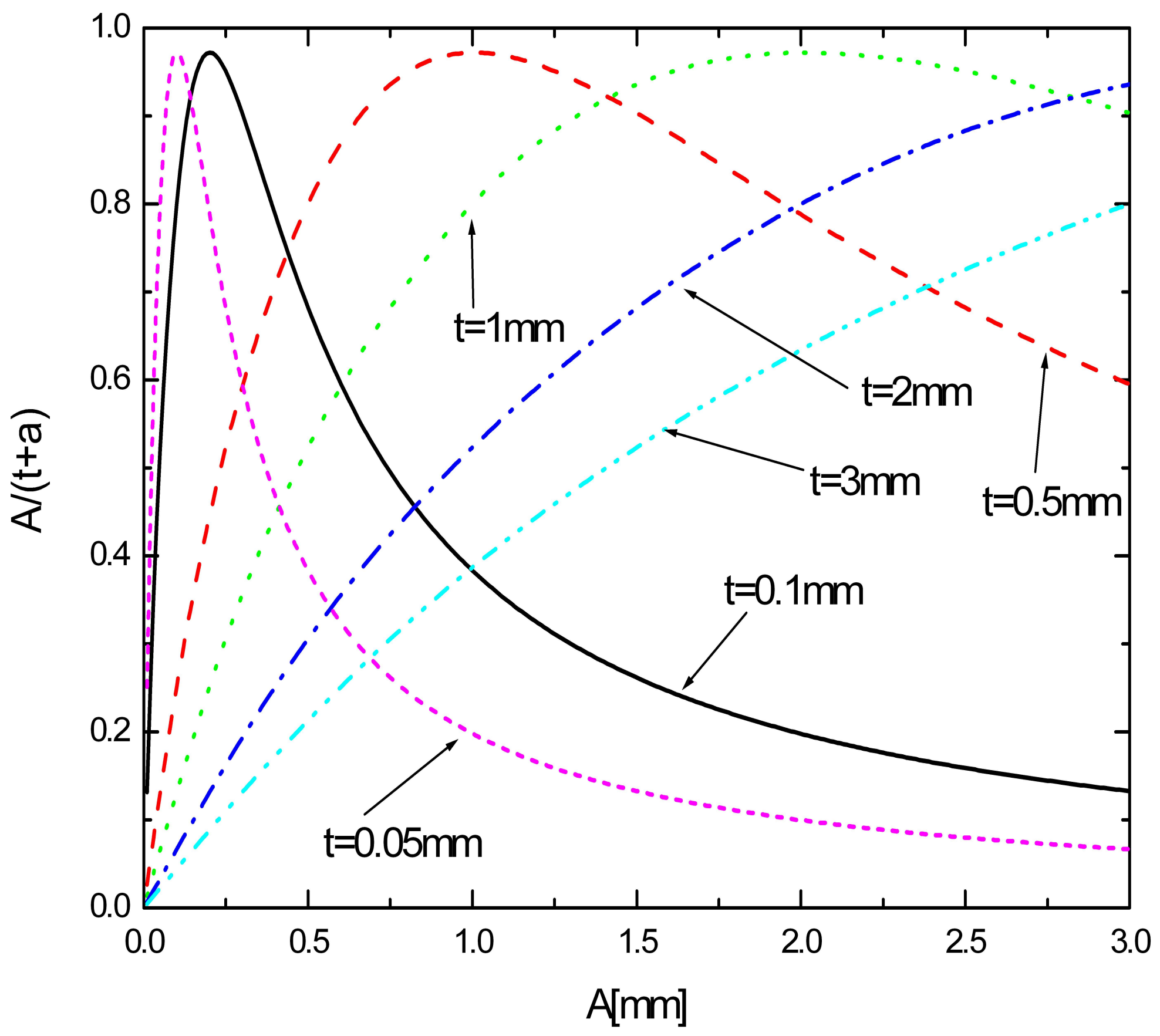


Front lens capsule

\section{Anterior chamber}

\section{Rear lens capsule}




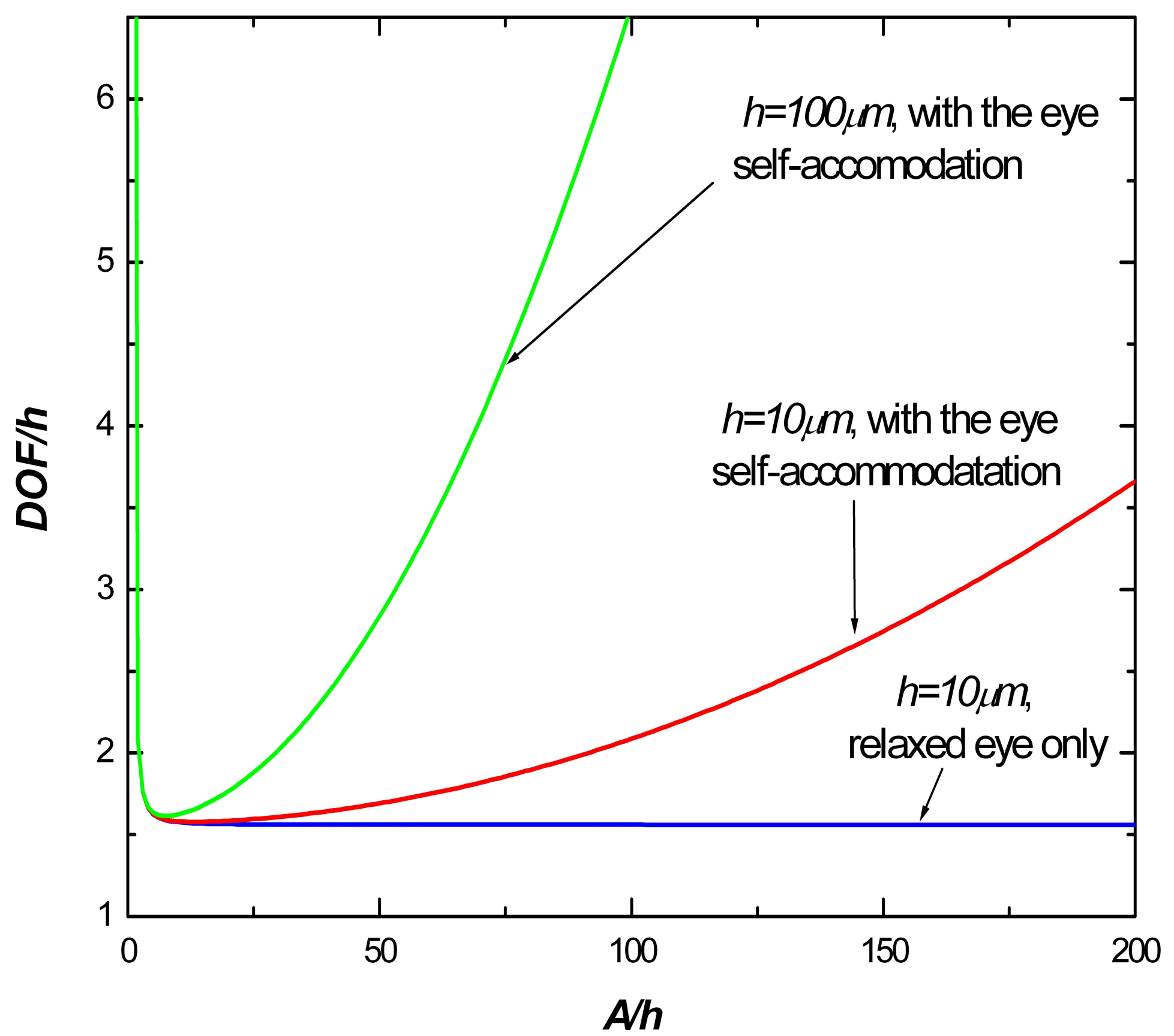

